\title{
Characteristics of Ultraviolet Photodetector Based on Li-doped ZnO Nanorods
}

\author{
Chun-Chi Chou, ${ }^{1 *}$ Li-Hsing Shih, ${ }^{1}$ and Shoou-Jinn Chang ${ }^{2}$ \\ ${ }^{1}$ Department of Resources Engineering, National Cheng Kung University, Tainan 701, Taiwan \\ ${ }^{2}$ Institute of Microelectronics and Department of Electrical Engineering, \\ Center for Micro/Nano Science and Technology, Advanced Optoelectronic Technology Center, \\ National Cheng Kung University, Tainan 701, Taiwan
}

(Received January 3, 2020; accepted June 3, 2020)

Keywords: zinc oxide, nanorod, hydrothermal, photodetectors

In this study, we report one-dimensional Li-doped $\mathrm{ZnO}$ nanorods (NRs) fabricated on glass substrates by the hydrothermal method and the fabrication of Li-doped ZnO NR photodetectors. It was found that the as-grown Li-doped $\mathrm{ZnO} \mathrm{NRs}$ were structurally equivalent to a wurtzite structure. The NRs had average diameters of $\sim 125, \sim 138$, and $\sim 176 \mathrm{~nm}$. It was seen clearly that the Li-doped $\mathrm{ZnO} \mathrm{NRs}$ had a uniform hexagonal structure. The on/off current contrast ratio of the Li-doped $\mathrm{ZnO} \mathrm{NR}$ photodetector was approximately 19.3, and its dynamic response was revealed to be reproducible and stable.

\section{Introduction}

One-dimensional $\mathrm{ZnO}$ nanostructures endow remarkable chemical and physical attributes to optoelectronic and electronic devices, and have therefore attracted an enormous amount of attention. As is well known, $\mathrm{ZnO}$ is a II-VI semiconductor with a large exciton binding energy of $60 \mathrm{meV}$ and a wide band gap of $3.37 \mathrm{eV}$ at room temperature. ${ }^{(1)} \mathrm{ZnO}$ has the hexagonal wurtzite structure with the lattice constants $a=3.24 \AA$ and $c=5.20 \AA$, and usually demonstrates n-type semiconductivity. ${ }^{(2)}$ Without sacrificing its high crystallinity, $\mathrm{ZnO}$ can be synthesized by many methods and used in various nanostructures. The $\mathrm{ZnO}$ grown with high quality has various morphologies, such as nanorod (NR), nanodisc, nanoneedle, nanotube, nanosheet, and nanowire. ${ }^{(3-5)} \mathrm{ZnO}$ has been widely used in different applications, such as ultraviolet photodetectors, dye-sensitized solar cells, surface acoustic wave devices, gas sensors, light-emitting diodes, varistors, laser diodes, field emission devices, and optical resonators. ${ }^{(6-9)} \mathrm{A} \mathrm{ZnO} \mathrm{NR}$ is an example of a tunable one-dimensional nanostructure with a high aspect ratio, made from a single $\mathrm{ZnO}$ crystal elongated along the $c$-axis, making it highly mechanically stable. $\mathrm{ZnO}$ nanostructure synthesis by the hydrothermal method exhibits distinct advantages, such as uniform morphology, high yield, controllable and simple fabrication process, homogeneous size, low cost, and low temperature features. ${ }^{(10-14)}$ In addition, in the hydrothermal method, all wastes are confined to the solutions, facilitating their treatment ${ }^{*}$ Corresponding author: e-mail: ncku@jsoon.com https://doi.org/10.18494/SAM.2020.2814 
and making the method environmentally benign and applicable to large areas; therefore, it is cost-effective.

The $\mathrm{ZnO}$ NR photodetectors have a much higher photocurrent than the conventional $\mathrm{ZnO}$ film photodetectors. The reasons are as follows. First, $\mathrm{ZnO}$ NR structures can increase the amount of light trapped by reflection and absorption. Consequently, the light trapping efficiency under light illumination is improved because of increased photocurrent efficiency and photon path length by the scattering of incident light between the freestanding NRs. Moreover, each $\mathrm{ZnO}$ freestanding NR can accelerate oxygen desorption and adsorption. ${ }^{(15,16)}$ In the dark, the oxygen molecules adsorb onto the ZnO NR surface by capturing free electrons. Upon irradiating UV light, oxygen molecules desorb from the surface, leading to an increased free carrier concentration and a reduced Schottky barrier height. ${ }^{(17,18)}$

Zinc oxide nanostructures doped with various elements are studied extensively because such doping can improve the nanostructure properties. In addition, transition-metal doping into $\mathrm{ZnO}$ can change many properties, such as surface morphology and structural, electrical, and optical properties. Theoretically, substituting Group I elements into Zn sites results in p-type conductivity. Unfortunately, p-type $\mathrm{ZnO}$ is very difficult to make because of low solubility limits, the existence of various naturally occurring or spontaneously generated donor defects such as zinc interstitials or zinc vacancies, and self-compensation. Among Group I elements, Li is the best candidate because the size of $\mathrm{Li}$ ions $(0.060 \mathrm{~nm})$ is close to that of $\mathrm{Zn}$ ions $(0.074 \mathrm{~nm})$, enabling $\mathrm{Li}$ ions to easily substitute into $\mathrm{Zn}$ sites. According to previous literature, the content of the Group I element Li substituting for $\mathrm{Zn}$ can be as high as 30 at.\%, and its effect on the $\mathrm{ZnO}$ physical properties is significant. According to previous literature, Li substitution into $\mathrm{Zn}$ sites leads to p-type conductivity because the various naturally occurring or spontaneously generated donor defects such as zinc interstitials, zinc vacancies, and oxygen vacancies can be suppressed. Various physical vapor deposition (PVD) methods have been reported for depositing $\mathrm{ZnO}$, such as PLD, sputtering, and ion beam deposition, but only the sputtering method can generate a surface having a morphology close to that of a single crystal on an amorphous or low-temperature substrate. ${ }^{(19-22)}$

In this study, we address the synthesis of Li-doped ZnO NRs on a glass substrate by a radio frequency (RF)-magnetron-sputtering-assisted low-temperature hydrothermal method and the composition of NRs prepared at temperatures of 70,80 , and $90{ }^{\circ} \mathrm{C}$. We investigate the structural, morphological, and optical properties of the samples. In addition, we demonstrate the suppression of various naturally occurring or spontaneously generated donor defects and the fabrication of a photodetector sensor by the thermal evaporation method. All the devices are fabricated in a test chamber at room temperature. The test devices are then illuminated with UV light, which has physical and electrooptical properties.

\section{Experiment}

First, prior to the growth of a zinc oxide seed layer, we cleaned the glass substrate by immersing it in acetone and isopropyl alcohol for $10 \mathrm{~min}$, followed by rinsing with deionized water, and finally drying with flowing nitrogen. A 25-nm-thick $\mathrm{ZnO}$ seed layer was then 
deposited on the glass substrate by RF magnetron sputtering. Then, the Li-doped ZnO NR arrays were formed by the hydrothermal method using lithium nitrate $\left(\mathrm{Li}\left(\mathrm{NO}_{3}\right)_{2}\right)$, zinc nitrate hexahydrate $\left(\mathrm{Zn}\left(\mathrm{NO}_{3}\right)_{2} \cdot 6 \mathrm{H}_{2} \mathrm{O}\right)$, and hexamethylenetetramine $\left(\mathrm{C}_{6} \mathrm{H}_{12} \mathrm{~N}_{4}\right.$, HMTA) as raw materials in a 0.1:0.9:1 ratio with a controlled concentration of $0.1 \mathrm{M}$ for $8 \mathrm{~h}$ at 70,80 , and $90{ }^{\circ} \mathrm{C}$.

The Bruker D8 X-ray diffractometer (XRD) was used to measure and examine the crystal structures of the pure $\mathrm{ZnO}$ and $\mathrm{Li}$-doped $\mathrm{ZnO}$ NRs. A field electron emission scanning electron microscopy device (FE-SEM, Hitachi S-4800I) was used to measure and examine the surface morphologies of pure $\mathrm{ZnO}$ and Li-doped $\mathrm{ZnO}$ NRs. To fabricate the photodetector with Li-doped $\mathrm{ZnO}$ NRs, a thick Au film was deposited on the $\mathrm{ZnO}$ seed layer as a contact electrode through an interdigitated shadow mask using an E-beam gun system. Figure 1 shows a schematic diagram of the fabricated Li-doped ZnO NR photodetector. The current-voltage $(I-V)$ characteristics of the fabricated Li-doped $\mathrm{ZnO}$ NR photodetectors were measured in the dark and under illumination using a Keithley 237 semiconductor parameter analyzer.

\section{Results and Discussion}

Figures 2(a)-2(c) show top-view FESEM images of the Li-doped ZnO NRs grown at 70, 80, and $90^{\circ} \mathrm{C}$, respectively. As shown in Fig. 2, high-uniform-distribution Li-doped ZnO NRs were successfully grown on the glass substrate. During the synthesis, $\operatorname{Li}\left(\mathrm{NO}_{3}\right)_{2}, \mathrm{HMTA}$, and $\mathrm{Zn}\left(\mathrm{NO}_{3}\right)_{2}$ act as a $\mathrm{pH}$ buffer that keeps the $\mathrm{pH}$ constant and $\mathrm{Li}$ and $\mathrm{Zn}$ ion sources, respectively. It was found that the NRs have average diameters of $\sim 125, \sim 138$, and $\sim 176 \mathrm{~nm}$. It can be seen clearly that the Li-doped $\mathrm{ZnO}$ NRs have a uniform hexagonal structure.

Figure 3 shows the measured XRD spectra of the Li-doped ZnO NRs grown at different temperatures. The peaks of all samples are attributed to the (002) and (004) diffractions of $\mathrm{ZnO}$ with a wurtzite structure. The small linewidths observed in the XRD spectrum of the Li-doped $\mathrm{ZnO}$ NRs indicate a high-crystalline-quality wurtzite structure, no impurities, and the complete transformation of precursors into the $\mathrm{ZnO}$ phase. This result suggests that the $\mathrm{Li}$-doped $\mathrm{ZnO}$ NRs are preferentially oriented in the (002) crystal plane along the $c$-axis.

Figure 4 shows the photoresponse cycle of a Li-doped $\mathrm{ZnO}$ NR photodetector measured three times. The on/off current contrast ratio of the Li-doped $\mathrm{ZnO}$ NR photodetector is approximately 19.3, and the dynamic response is reproducible and stable. The photocurrent of the Li-doped $\mathrm{ZnO} \mathrm{NR}$ photodetector increases exponentially until saturation, and the current slowly decreases after turning off the UV source. This decrease is associated with the surface-defect-related carrier trapping centers. The surface-defect-related carrier trapping

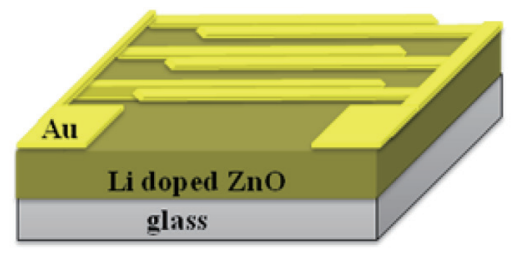

Fig. 1. (Color online) Schematic diagram of the fabricated Li-doped $\mathrm{ZnO}$ NR photodetector. 
centers retard the charge carrier collection and charge carrier recombination speeds when the UV light is turned on or off. The structure of the NRs also increases the efficiency of light trapping during illumination. When the light is incident on the NRs, the photon path length and photocurrent efficiency increase, which increases the amount of light that is trapped by scattering between free-standing NRs. This indicates that the Li-doped ZnO NR device has potential for use in UV light sensing. ${ }^{(23-25)}$

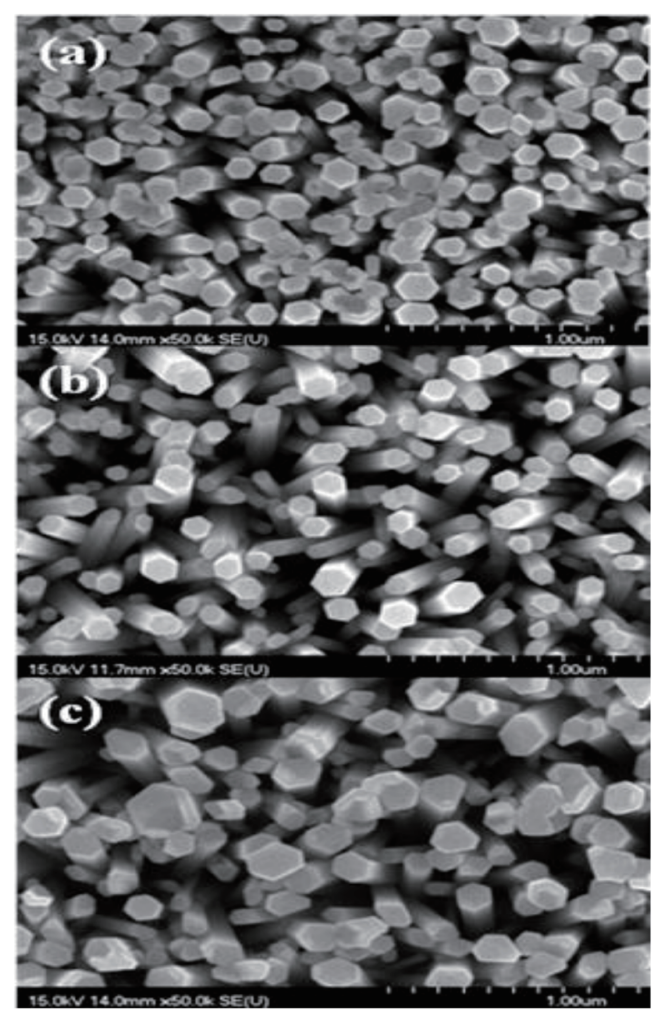

Fig. 2. Top-view FESEM images of the Li-doped ZnO NRs grown at (a) 70, (b) 80, and (c) $90{ }^{\circ} \mathrm{C}$.

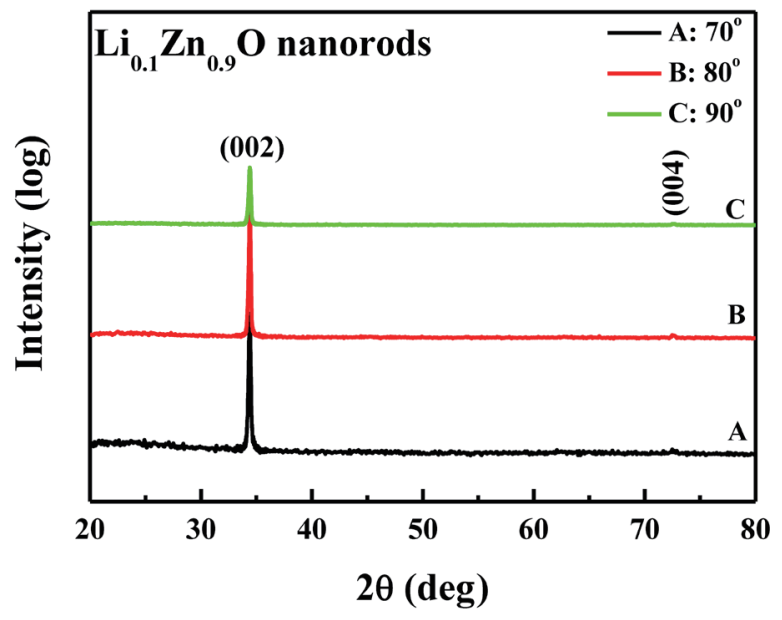

Fig. 3. (Color online) XRD spectra of Li-doped $\mathrm{ZnO}$ NRs grown at 70,80 , and $90^{\circ} \mathrm{C}$. 


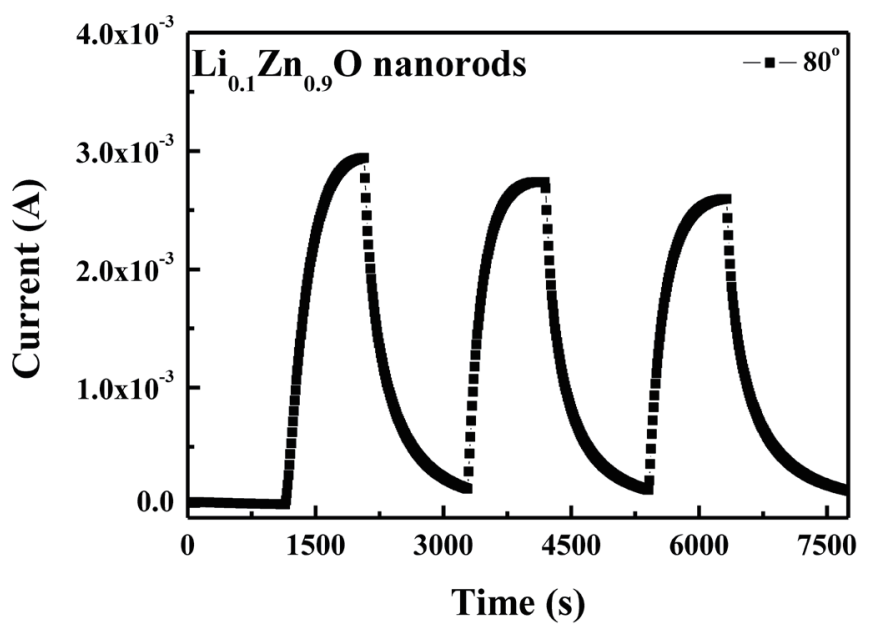

Fig. 4. Transient switching photoresponse curve of Li-doped $\mathrm{ZnO}$ NR photodetector as the UV excitation was switched on and off.

\section{Conclusions}

We reported the successful preparation of Li-doped $\mathrm{ZnO}$ NRs on a glass substrate by the hydrothermal method at 70,80 , and $90{ }^{\circ} \mathrm{C}$. The Li-doped $\mathrm{ZnO}$ NRs grown at $80{ }^{\circ} \mathrm{C}$ had the least number of defects and oxygen vacancies. SEM observations revealed that Li-doped $\mathrm{ZnO}$ NRs were successfully evenly grown at a high density on the glass substrate with the NRs being mostly perpendicular to the surface of the substrate. The on/off current contrast ratio of the Li-doped $\mathrm{ZnO}$ NR photodetector was approximately 19.3. The results indicated that the transient response of the Li-doped $\mathrm{ZnO}$ NR photodetector is stable and reproducible.

\section{References}

1 C. B. Yao, K. X. Zhang, X. Wen, J. Li, Q. H. Li, and S. B. Yang: J. Alloys Compd. 698 (2017) 284. https://doi. org/10.1016/j.jallcom.2016.12.158

2 S. J. Young: IEEE J. Sel. Top. Quantum Electron. 20 (2014) 3801204. https://doi.org/10.1109/ JSTQE.2014.2316599

3 S. J. Chang, B. G. Duan, C. H. Hsiao, C. W. Liu, and S. J. Young: IEEE Photonics Technol. Lett. 26 (2014) 66. https://doi.org/10.1109/LPT.2013.2289322

4 Y. H. Liu, S. J. Young, C. H. Hsiao, L. W. Ji, T. H. Meen, W. Water, and S. J. Chang: IEEE Photonics Technol. Lett. 26 (2014) 645. https://doi.org/10.1109/LPT.2014.2301845

5 K. J. Chen, F. Y. Hung, S. J. Chang, S. J. Young, and Z. S. Hu: Curr. Appl. Phys. 11 (2011) 1243. https://doi. org/10.1016/j.cap.2011.03.033

6 Y. X. Liang, S. Q. Fan, X. D. Chen, and Y. T. Hu: J. Nanotechnol. 9 (2018) 1917. https://doi. org/10.3762\%2Fbjnano.9.183

7 S. J. Young and Y. H. Liu: Microelectron. Eng. 148 (2015) 14. https://doi.org/10.1016/j.mee.2015.07.009

8 C. W. Liu, S. J. Chang, C. H. Hsiao, K. Y. Lo, T. H. Kao, B. C. Wang, S. J. Young, K. S. Tsai, and S. L. Wu: IEEE J. Sel. Top. Quantum Electron. 20 (2014) 3800707(1-7). https://doi.org/10.1109/JSTQE.2014.2312935

9 T. P. Chen, S. J. Young, S. J. Chang, C. H. Hsiao, and S. L. Wu: IEEE Trans. Electron Devices 60 (2013) 229. https://doi.org/10.1109/TED.2012.2228655

10 S. J. Young and Y. H. Liu: IEEE J. Sel. Top. Quantum Electron. 23 (2017) 3800905. https://doi.org/10.1109/ JSTQE.2017.2684540 
11 J. Kwon, S. Hong, G. Kim, Y. D. Suh, H. Lee, S. Y. Choo, D. Lee, H. Kong, J. Yeo, and S. H. Ko: Appl. Surf. Sci. 447 (2018) 1. https://doi.org/10.1016/j.apsusc.2018.03.155

12 Y. C. Chang, C. C. Hsu, S. H. Wu, K. W. Chuang, and Y. F Chen: Appl. Surf. Sci. 447 (2018) 213. https://doi. org/10.1016/j.apsusc.2018.03.240

13 S. J. Young and Y. H. Liu: IEEE Trans. Electron Devices 63 (2016) 3160. https://doi.org/10.1109/ TED.2016.2582506

14 S. J. Lei, C. J. Tao, J. L. Li, X. Zhao, and W. Z. Wang: Appl. Surf. Sci. 452 (2018) 148. https://doi.org/10.1016/ j.apsusc.2018.05.005

15 Y. Cho, H. Ji, H. Kim, J. Yoon, and B. Choi: J. Nanosci. Nanotechnol. 18 (2018) 5996. https://doi.org/10.1166/ jnn.2018.15594

16 D. Calestani, M. Culiolo, M. Villani, D. Delmonte, M. Solzi, T. Y. Kim, S. W. Kim, L. Marchini, and A. Zappettini: Nanotechnology 29 (2018) 596. https://doi.org/10.1088/1361-6528/aac850

17 M. Yu, Y. X. Ma, J. H. Liu, X. J. Li, S. M. Li, and S. Y. Liu: Appl. Surf. Sci. 390 (2016) 266. https://doi. org/10.1016/j.apsusc.2016.08.061

18 S. J. Young and Y. H. Liu: Sens. Actuators, A 269 (2018) 363. https://doi.org/10.1016/j.sna.2017.11.044

19 C. Zhang, X. H. Huang, H. F. Liu, S. J. Chua, and C. A. Ross: Nanotechnology 27 (2016) 73. https://doi. org/10.1088/0957-4484/27/48/485604

20 S. J. Young and K. W. Yuan: IEEE Trans. Electron Devices 66 (2019) 3978. https://doi.org/10.1109/ TED.2019.2926021

21 L. W. Ji, Y. J. Hsiao, S. J. Young, W. S. Shih, W. Water, and S. M. Lin: IEEE Sens. J. 15 (2015) 762. https://doi. org/10.1109/JSEN.2014.2353630

22 S. J. Young and L. T. Lai: IEEE Trans. Electron Devices 67 (2020) 304. https://doi.org/10.1109/ TED.2019.2946931

23 Y. H. Liu, S. J. Young, L. W. Ji, and S. J. Chang: IEEE Trans. Electron Devices 62 (2015) 2033. https://doi. org/10.1109/TED.2015.2416730

24 S. J. Young and L. T. Lai: Microelectron. Eng. 148 (2015) 40. https://doi.org/10.1016/j.mee.2015.07.012

25 Y. Zhou, X. Li, H. Y. Yu, G. L. Hu, and J. M. Yao: J. Nanopart. Res. 18 (2016) 359. https://doi.org/10.1007/ s11051-016-3678-5 